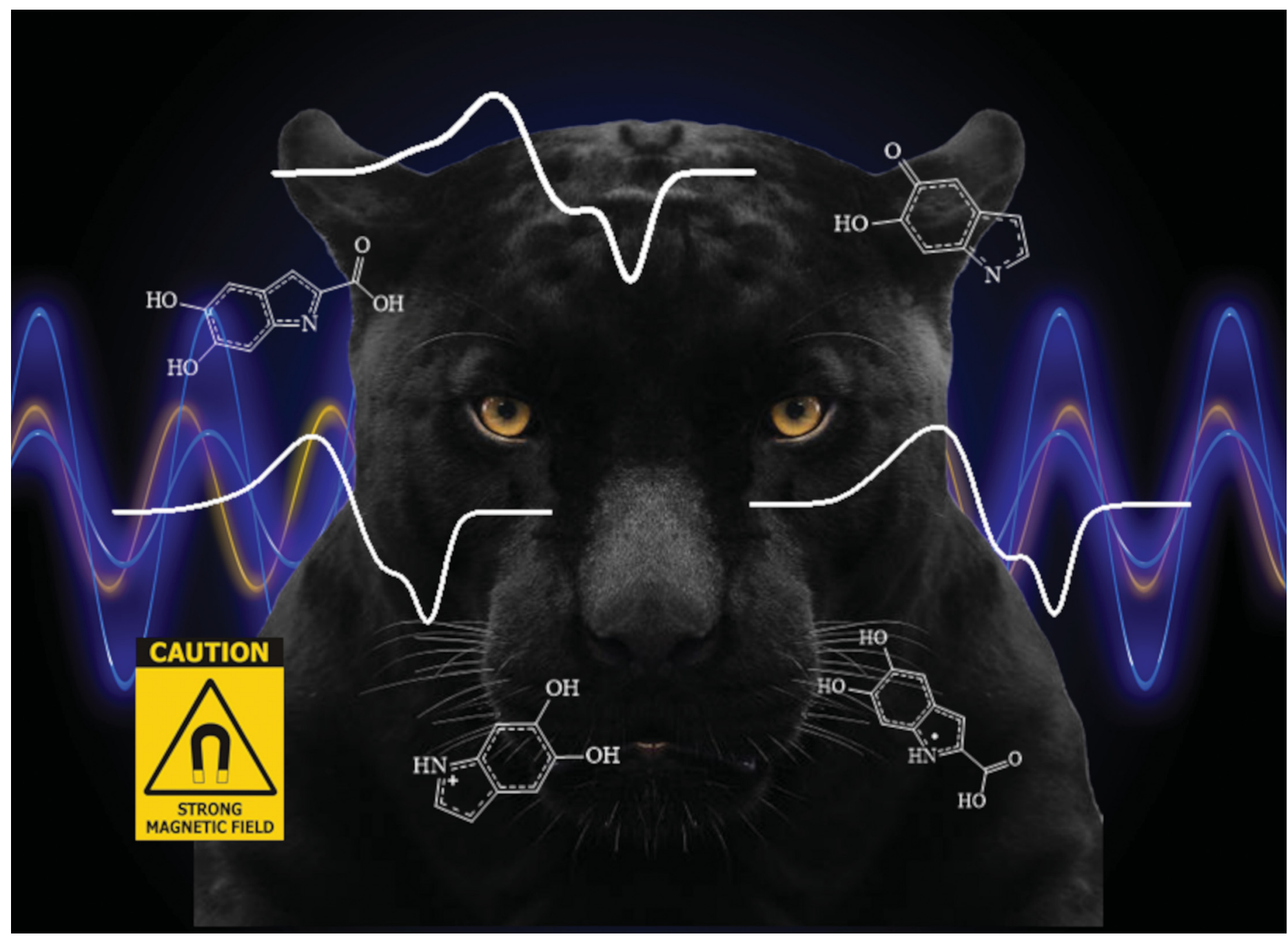

Showcasing research from Professor Carlos F. O. Graeff's laboratory, School of Science, São Paulo State University, Bauru/São Paulo, Brazil.

High-field/high-frequency EPR spectroscopy on synthetic melanin: on the origin of carbon-centered radicals

Melanin is a promising material at the helm of bioelectronic applications due to its inherent biocompatibility and hydration-dependence conductivity. Additionally, its free-radical species can be considered a potential charge carrier or an indirect spin-label for charge transport. Hence, by applying high-field/high-frequency EPR spectroscopy, information regarding the intrinsic melanin radical's possible nature, i.e., carbon-centered radicals (CCR), was obtained. We have found that at least four different monomeric structures should be evoked for the CCR EPR signal.
As featured in:

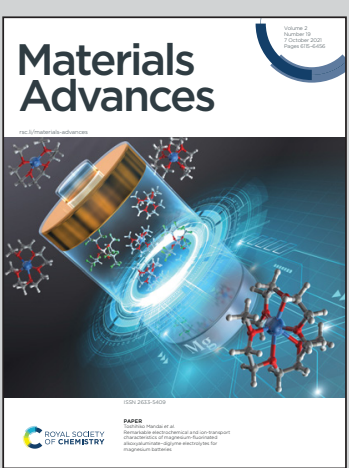

See C. F. O. Graeff et al.,

Mater. Adv., 2021, 2, 6297. 
Check for updates

Cite this: Mater. Adv., 2021, 2, 6297

Received 18th May 2021,

DOI: $10.1039 / \mathrm{d} 1 \mathrm{ma} 00446 \mathrm{~h}$

rsc.li/materials-advances Accepted 26th July 2021

\section{High-field/high-frequency EPR spectroscopy on synthetic melanin: on the origin of carbon- centered radicals $\uparrow$}

\author{
J. V. Paulin, (D) ${ }^{a}$ A. Batagin-Neto, (DD ${ }^{\text {ab }}$ B. Naydenov, (D) ${ }^{c}$ K. Lips (iD ${ }^{\text {cde }}$ and \\ C. F. O. Graeff iD *af
}

\begin{abstract}
Among all the pigments found in nature, melanin has drawn considerable attention for bioelectronics applications as it contains a set of physicochemical properties such as antioxidant activity, metal chelation, biocompatibility, broad-band absorbance throughout the UV-visible region, hydration-dependent conductivity and intrinsic and extrinsic free radical character. A better understanding of these properties is a matter of significant importance to improve melanin-based organic devices efficiently. Therefore, the origin of melanin free-radicals was studied using continuous-wave electron paramagnetic resonance (EPR) spectroscopy at $263 \mathrm{GHz}$ for standard nonfunctionalized and sulfonated derivatives. Our results indicated that more than one radical species should be evoked to study the free-radical nature of standard and sulfonated melanin. Additionally, we also confirm that the free-radical system of sulfonated melanin is similar to non-functionalized melanin. Based on the $g$-anisotropy analysis, we propose which potential units could be responsible for carbon-centered radicals.
\end{abstract}

\section{Introduction}

Melanins are natural pigments with several functions ranging from aesthetic, ${ }^{1}$ antimicrobial ${ }^{2}$ and antioxidant ${ }^{3,4}$ behaviors to radical scavenging ${ }^{4}$ and photo $^{5}$ and radioprotection. ${ }^{6}$ Furthermore, it also has a series of physicochemical properties such as biocompatibility, ${ }^{7}$ broad-band UV-vis absorbance, ${ }^{8}$ paramagnetism $^{9}$ and conductivity depending on the hydration. ${ }^{10-12}$ Due to these properties, melanin has generated significant interest to be employed in a broad range of technological applications. ${ }^{13-25}$

Melanin is a macromolecular system consisting of 5,6dihydroxyindole (DHI) and 5,6-dihydroxyindole-2-carboxylic acid (DHICA) monomers and their different redox states (see Fig. 1(a)). ${ }^{7}$ These monomers react with one another to form small oligomers that then self-assemble in $\pi$ - $\pi$-stacked

\footnotetext{
${ }^{a}$ São Paulo State University (UNESP), School of Sciences, Postgraduate Program in Science and Technology of Materials (POSMAT), Bauru, Brazil. E-mail: carlos.graeff@unesp.br

${ }^{b}$ São Paulo State University (UNESP), Campus of Itapeva, Itapeva, Brazil ${ }^{c}$ Helmholtz-Zentrum Berlin für Materialien und Energie GmbH, Berlin, Germany

${ }^{d}$ Freie Universität Berlin, Department Physics, Berlin, Germany

${ }^{e}$ University of Utah, Department of Physics and Astronomy, Salt Lake City, USA ${ }^{f}$ São Paulo State University (UNESP), School of Sciences, Department of Physics, Bauru, Brazil

$\dagger$ Electronic supplementary information (ESI) available: Different structural units used for DFT calculations; additional EPR analyses; spin density maps; phase correction procedure. See DOI: 10.1039/d1ma00446h
}

supramolecular units of different sizes with an interplanar spacing of $3.7 \AA^{7}$

Perhaps one of the most studied properties of melanin is its persistent paramagnetic signal, which can easily be detected with continues-wave (CW) electron paramagnetic resonance (EPR), under various experimental conditions. ${ }^{28-46}$ Nowadays, it is accepted that the melanin paramagnetic system consists of one

(a)<smiles>Oc1cc2cc[nH]c2cc1O</smiles><smiles>O=C[18OH]</smiles><smiles>[R]C1=CC2=CC(=O)C(O)=CC2=N1</smiles>
(QI)<smiles>[R]C1=[N+]2C=C(C=O)C(=O)C=C2C=C1</smiles>
(SQ)

(b)<smiles>[R]Oc1cc2cc([R])n([R3])c2cc1O[R20]</smiles><smiles></smiles>

(IQ)<smiles>[R]c1cc2cc(O)c(O)cc2[nH]1</smiles>

Fig. 1 (a) The building block monomers for non-functionalized melanin. $H Q$ denotes hydroquinone, $Q$ I quinone imine, IQ indolequinone and $S Q$ semiquinone. $\mathrm{Ndef}^{+}$is attributed to a charged structure, a synthesis defect. ${ }^{26}$ (b) Proposed building blocks of sulfonated melanin. ${ }^{27}$ 


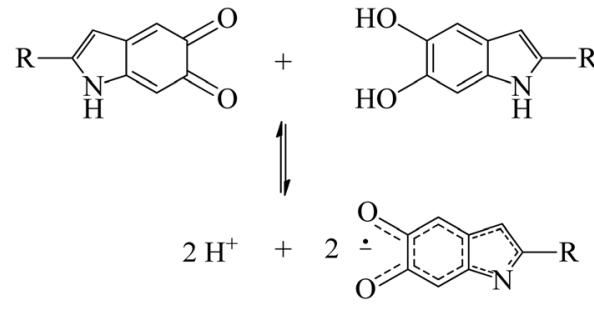

Scheme 1 The comproportionation equilibrium reaction. Indolequinone and hydroquinone moieties interact together to obtain negatively charged semiquinones and protons.

intrinsic free-radical originated from the growth of its macrostructure and an extrinsic free-radical usually associated to a molecular probe of the redox state of the melanin monomer. ${ }^{30,42,47,48}$ The concentration of the extrinsic free radicals is determined by the comproportionation equilibrium reaction, ${ }^{39,44}$ Scheme 1 , and has played a significant role in developing the current understanding of some basic physicochemical properties of melanin. ${ }^{7,36,47,49}$ The intrinsic radical is defined as carbon-centered radicals (CCR); specifically, its spin density is located in carbon atoms; the extrinsic one is semiquinone-free radicals (SFR), i.e., the spin density is found in oxygen atoms. ${ }^{34}$ Furthermore, $g$-values (around 2.0030 for CCR and 2.0050 for SFR) and line shape can be used to distinguish these species. ${ }^{28,34,43,44,49}$

At present, there are relatively few reports in the literature trying to understand the origin of melanin EPR spectra, especially at high magnetic fields. ${ }^{29,34,37,38,46}$ For instance, Q-band $(v=$ $35 \mathrm{GHz}, B_{0} \approx 1.25 \mathrm{~T}$ ) EPR spectra recorded over a range of $\mathrm{pH}$ for different melanins demonstrate the presence of anionic and neutral radicals with different $g$-values. ${ }^{29} \mathrm{~A}$ few years later, the analysis of the Q-band spectra was refined using computer simulation analysis. The existence of four species was proposed and they were related to cation radicals with extended delocalization of their unpaired spins and anion $o$-semiquinone radicals with relatively localized unpaired electrons. ${ }^{46}$ In previous work, we used DFT $^{49}$ and X-band $(v=9.7 \mathrm{GHz})$ EPR spectral simulations ${ }^{37}$ to hypothesize that CCR could be ascribed to NDef $^{+}$structures and SFR to anionic indolequinone and semiquinone radicals.

High-field/high-frequency EPR (HFEPR) spectroscopy has high sensitivity towards small molar spin concentrations and spin systems that cannot be observed at low frequencies. ${ }^{50-53}$ It can enhance the signal resolution of the principal values of the $\boldsymbol{g}$ tensor ( $g$-anisotropy) to better distinguish and characterize the paramagnetic species. Therefore, with the rapid evolution of EPR spectroscopy in recent times, ${ }^{52,53}$ it is time to take advantage of the higher spectral resolution of the HFEPR and study melanin's free-radical nature. Building upon our previous EPR work, we applied $263 \mathrm{GHz}$ EPR spectroscopy to different synthetic non-functionalized (NF) and sulfonated (S) melanin derivatives. Sulfonated melanin, whose basic structure is shown in Fig. 1(b), behaves closely to standard melanin at X-band CW EPR ${ }^{38}$ and has promising properties for bioelectronics applications. ${ }^{17,54-57}$ The experimental results were interpreted with spectral simulations and density functional theory, throwing light on the $g$-anisotropy of melanin-based radicals and their possible origin.

\section{Materials and methods}

\section{Melanin synthesis}

Non-functionalized melanin was synthesized by dissolving $0.3 \mathrm{~g}$ of DL-DOPA (Sigma-Aldrich, 98\%) in $60 \mathrm{~mL}$ of MiliQ water (18 $\mathrm{M} \Omega \mathrm{cm}$ ) and $400 \mu \mathrm{L}$ of ammonium hydroxide (Synth, 28$30 \%) .{ }^{58}$ Ammonium hydroxide was used to increase the $\mathrm{pH}$ solution to $8-9$, the melanogenesis condition. ${ }^{3}$ The synthesis mixture was stirred at room temperature $(T \approx 300 \mathrm{~K})$ and oxygenated with an air pump for three days (NFMel) or in a $150 \mathrm{~mL}$ stainless steel reactor with $6 \mathrm{~atm}$ internal pressure of industrial oxygen gas for 6 hours (NFMel-P). Afterward, the solution was filtered using a 3500 MWCO dialysis membrane for four days. The dialysate medium was MiliQ water and it was changed every day. After drying the suspended colloidal particles for two days at $353 \mathrm{~K}$ in an oven, a black powder was obtained. These different samples have identical polymerization structures but different oxidation degrees (NFMel < NFMel-P).

Sulfonated-functionalized melanin derivative (SMel) was obtained by the oxidation of $1.50 \mathrm{~g}$ of DL-DOPA in $200 \mathrm{~mL}$ of DMSO (PA, Vetec, 99.9\%) with $0.93 \mathrm{~g}$ of benzoyl peroxide (Vetec, 75.0-80.0\%). ${ }^{57,59}$ The mixture was kept under magnetic stirring for 58 days at room temperature $(T \approx 300 \mathrm{~K})$. Next, the solution was heated at $413 \mathrm{~K}$ to evaporate $3 / 4$ of the solvent and then mixed in acetonitrile (Synth, 99.5\%) and kept for two days. Afterward, the acetonitrile solution was centrifuged at $2500 \mathrm{rpm}$ for 15 minutes and the precipitate extracted was dried at $353 \mathrm{~K}$ in an oven.

All commercially available chemicals were used without further purification.

\section{HFEPR spectroscopy}

Continuous-wave (CW) HFEPR measurements were performed with a Bruker Elexsys 780 EPR quasi-optical spectrometer measuring the dispersion and absorption spectra simultaneously. The spectra were acquired with quadrature detection at $263 \mathrm{GHz}$ microwave frequency with $10 \mathrm{kHz}$ modulation frequency, $5 \mathrm{G}$ of modulation amplitude, $600 \mathrm{G}$ of sweep width and $0.1,1.0$ and $10.00 \mathrm{~mW}$ microwave powers. Trapped atomic nitrogen in the endohedral fullerene $\left(\mathrm{N} @ \mathrm{C}_{60}, g_{\text {iso }}=2.0020^{60}\right)$ was used as a reference for $g$-value determination.

The HFEPR spectra were processed by baseline and phase correction, ${ }^{61}$ obtained by adjusting the admixture of a linear combination between the absorption signal with its Hilbert transform until the integrated spectrum displayed a zero baseline (more details in ESI $\dagger$ ). The spectra were then analyzed using Easyspin (v.5.2.13) ${ }^{62}$ with the pepper routine with Gaussian function and anisotropic broadening.

\section{Electronic structure calculations}

For a deeper insight into the NF and S-melanin anisotropy, distinct charged and radicalar units based on the common building units (Fig. 1) of each melanin group were considered (see Fig. S1, ESI $\dagger$ ). The most stable structures were obtained by molecular dynamic calculations, as reported elsewhere. ${ }^{38}$ All the structures were pre-optimized in a Hartree-Fock approach using PM6 semiempirical approximation as implemented in 
the MOPAC2016 computational package. ${ }^{63}$ The most stable structures were then fully optimized in the framework of the density functional theory (DFT) using the B3LYP hybrid exchange-correlation functional and $6-31 \mathrm{G}(\mathrm{d}, \mathrm{p})$ basis set on all the atoms with the aid of GAMESS (NF-melanins) and Gaussian 09 (S-melanins) computational packages. ${ }^{64,65}$ EPR active compounds were designed by adding/removing one electron to/from the neutral structures. ${ }^{66,67}$ The charged units were then re-optimized with a DFT/B3LYP/6-31G(d,p) approach by considering the neutral optimized structures as starting geometries. For $\mathrm{Ndef}^{+}$, it was considered only its anionic structure (i.e., $\mathrm{Ndef}^{+}+\mathrm{e}^{-}=\mathrm{Ndef}$ ). The presence of $\mathrm{Ndef}^{+}$species may not be common; however, they arise from synthetic defectlike structures ${ }^{26}$ and are usually seen in the fitting of N1s XPS spectra. ${ }^{57,58,68,69}$ The $g$-values $\left(g_{x x}, g_{y y}\right.$ and $\left.g_{z z}\right)$ were estimated with the aid of an Orca 3.0.3 computational package, ${ }^{70}$ in a DFT/B3LYP/6-31G(d,p) approach.

\section{Results and discussion}

As mentioned in the introduction, most of the characterization of free-radical species in melanin was performed using X-band and more detailed information cannot be obtained due to the absence of resolved hyperfine coupling and the limited $\mathrm{g}$ resolution. Hence, for a complete hyperfine structure and $g$ anisotropy analysis, high-frequency EPR spectroscopy needs to be used. Fig. 2 presents the $263 \mathrm{GHz}$ HFEPR derivative absorption signal of $\mathrm{NF}$ and S-melanin at different microwave powers. All spectra show a rhombic character and no clear resolved hyperfine interaction (Fig. S2, ESI $\dagger$ ). Such features are different from the corresponding X-band spectra (see Fig. S3, ESI $\dagger$ ). The distortion of the melanin EPR spectra at higher microwave power can indicate that the paramagnetic system is composed of different species with similar $g$-values and different spin-lattice relaxation rates and consequently saturation effects. $^{34-36,38}$

The melanin free-radical system was evaluated at low microwave powers with EasySpin. ${ }^{62}$ We tested the hypothesis that the spectra are comprised of multiple resonant lines (one, two and three lines) with isotropic broadened (Fig. S4, ESI $\dagger$ ), anisotropic broadened with axial symmetry (Fig. S5, ESI $\dagger$ ), or rhombic symmetric lines (Fig. S6, ESI $\dagger$ ) with no success. The spectra were satisfactorily fitted assuming anisotropic broadened lines with rhombic symmetry and $g$-strain (Fig. S7, ESI $\dagger$ ). Such symmetry is different from earlier observations on Q-band EPR; ${ }^{46}$ however, it is commonly a good representation for indole materials ${ }^{71}$ and semiquinone radicals found in human nails. ${ }^{72}$ According to the last proposed simulation, the fit was relatively good in all cases; nonetheless, when three lines are
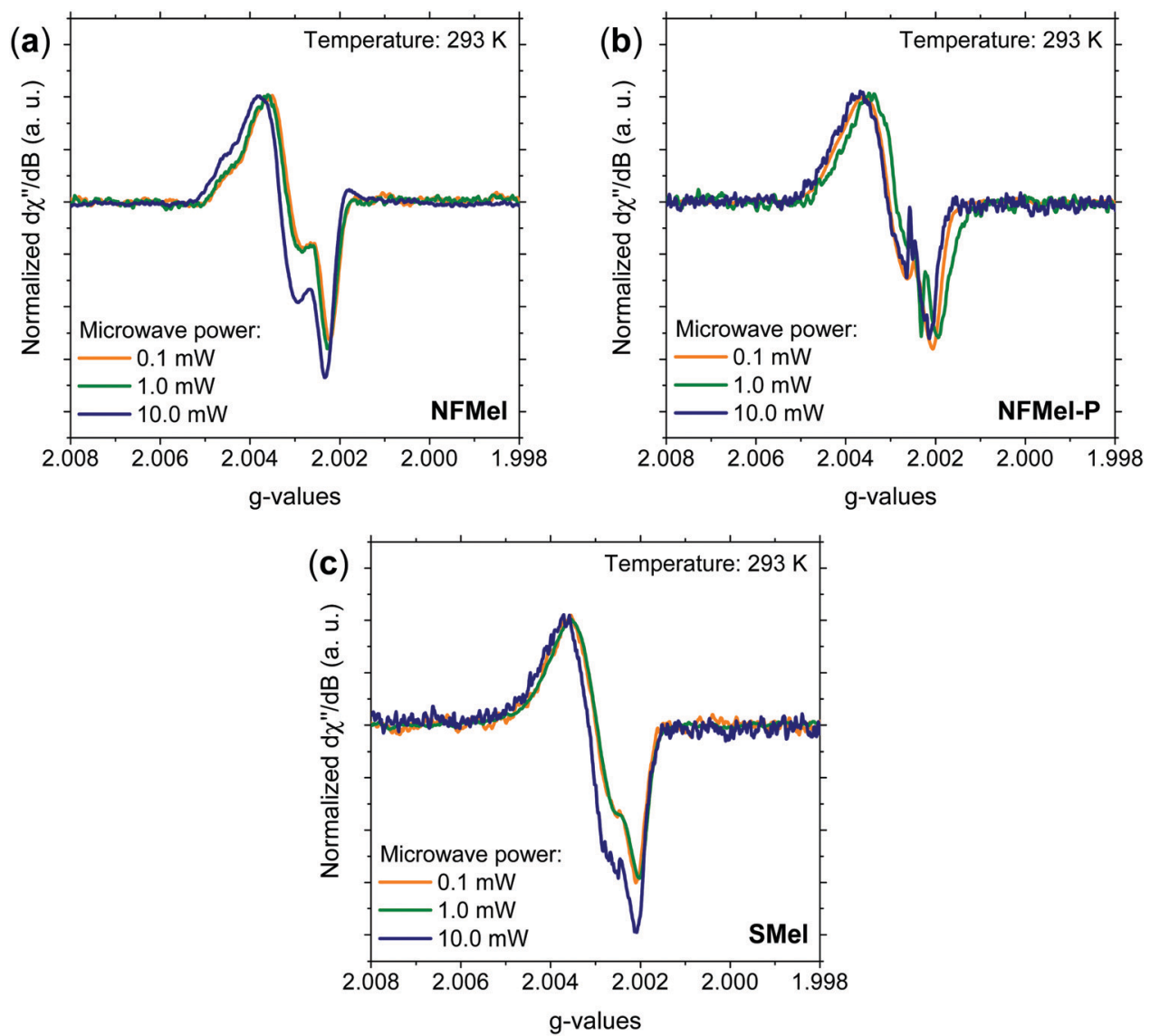

Fig. 2 Normalized $263 \mathrm{GHz}$ EPR derivative absorption spectra at $293 \mathrm{~K}$ and different microwave powers for (a) NFMel, (b) NFMel-P and (c) SMel. 


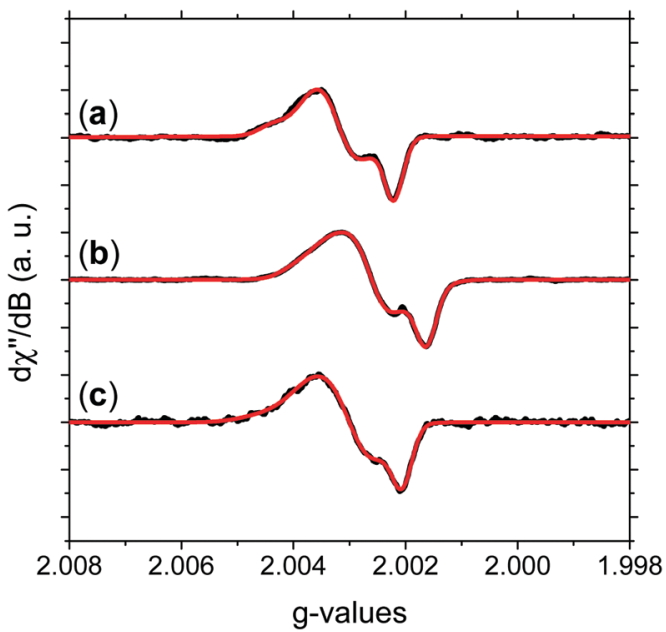

Fig. 3 Experimental HFEPR derivative absorption spectra (black line) and their fitting (red line) for (a) NFMel, (b) NFMel-P and (c) SMel at $0.1 \mathrm{~mW}$ microwave power. Fitting parameters are displayed in Table 1.

used, two of them have spectral features so similar that we could be just overparameterizing our analysis (Table S1, ESI $\dagger$ ). Therefore, we disregard such a possibility. On the other hand, we could not reproduce the shoulder at high g (Fig. S7a, ESI $\dagger$ ) with only one. Hence, we assumed that the spectra are composed of at least two different resonant lines. To further test this idea, we turned to the saturation phenomena mentioned before. The idea is that if the fitting lines do work at high microwave power spectra by changing only their intensity, our attribution is correct. In Fig. S8-S10 (ESI $\dagger$ ), we show that at $10 \mathrm{~mW}$ only with two resonant lines the fit is good and there is a relative variation in the spin density.

Fig. 3 shows the absorption HFEPR spectra and their respective fitting considering two paramagnetic species and rhombic symmetry for NFMel, NFMel-P and SMel. Moreover, from the fit shown in Fig. 3, the associated parameter values of each species converged to the values reported in Table 1. We also estimate the anisotropy of the $g$-tensor $(\Delta g)$, given by the difference between $g_{x x}$ and $g_{z z}$, which present greater precision than the absolute $g$-values ${ }^{73}$ and the spectrum rhombicity, calculated from $\left[\left(g_{x x}-g_{y y}\right) \cdot\left(g_{x x}-g_{z z}\right)^{-1}\right] \cdot^{71}$

We first take a look at the fitting analysis of NFMel. Our results show that this sample has an HFEPR spectrum with a

Table 1 Experimentally determined $g$-values for melanin derivatives

\begin{tabular}{|c|c|c|c|c|c|c|}
\hline \multirow[b]{2}{*}{ Component } & \multicolumn{2}{|l|}{ NFMel } & \multicolumn{2}{|c|}{ NFMel-P } & \multicolumn{2}{|l|}{$\underline{\text { SMel }}$} \\
\hline & A-I & A-II & B-I & B-II & C-I & C-II \\
\hline$g_{x x}$ & 2.0043 & 2.0037 & 2.0042 & 2.0038 & 2.0046 & 2.0038 \\
\hline$g_{y y}$ & 2.0030 & 2.0032 & 2.0032 & 2.0031 & 2.0033 & 2.0030 \\
\hline $\begin{array}{l}\delta y y \\
g_{z z}\end{array}$ & 2.0023 & 2.0022 & 2.0021 & 2.0021 & 2.0024 & 2.0021 \\
\hline$\sigma_{x x}\left(10^{-4}\right)$ & 5.10 & 5.71 & 5.00 & 9.58 & 6.98 & 8.61 \\
\hline$\sigma_{y y}\left(10^{-4}\right)$ & 9.99 & 4.83 & 8.77 & 6.00 & 2.27 & 6.54 \\
\hline$\sigma_{z z}\left(10^{-4}\right)$ & 0.02 & 0.01 & 5.46 & 2.32 & 2.03 & 1.60 \\
\hline$g_{\text {iso }}$ & 2.0032 & 2.0030 & 2.0032 & 2.0030 & 2.0034 & 2.0030 \\
\hline$\Delta g\left(10^{-5}\right)$ & 208 & 147 & 218 & 174 & 220 & 168 \\
\hline Rhombicity & 0.64 & 0.34 & 0.48 & 0.43 & 0.59 & 0.46 \\
\hline
\end{tabular}

low isotropic $g$-value $\left(g_{\text {iso }}<2.0035\right)$ and $g$-anisotropies $(\Delta g)$ of $208 \times 10^{-5}$ for species A-I and 147 for A-II. Such values indicate (i) the presence of CCR paramagnetic species and (ii) that the principal $g$-values of A-I can be fully resolved at the magnetic fields used for this study, whereas for A-II they are not resolved, i.e., there is an overlapping of $g_{x x}$ and $g_{y y}$.

For a $\pi$ radical, $g_{z z}$ usually has low spin-orbit coupling and is oriented perpendicularly to the aromatic system. ${ }^{74,75}$ In contrast, the in-plane orientations corresponding to $g_{x x}$ and $g_{y y}$ exhibit large shifts due to spin-orbit coupling originated from the radical structure and environment. ${ }^{74,75}$ The $g$-strain, the full width at half maximum of the Gaussian distributions of the main $g$-values, demonstrates low directional dependence at $g_{z z}$ and moderate to high at $g_{x x}$ and $g_{y y}$. This effect indicates that, although the species may be similar, they should present some local structural variation.

The $\boldsymbol{g}$ tensor of NFMel-P is similar to NFMel but has an increased directional dependence. This result indicates that NFMel and NFMel-P have similar free-radical species, as we have proposed in an earlier study. ${ }^{37,38}$ The different oxidation states of these samples and macrostructure are responsible for the differences.

To help with the interpretation, we analyze the $g$ tensor values obtained from the density functional theory of each anionic, cationic and radical structure summarized and shown in Fig. 4. Their colored spin density maps are shown in Fig. S11 and S12 (ESI $\dagger$ ).

Our earlier study showed that CCR species associated with $g_{\text {iso }}<2.0040$ would be compatible with anion and cation NFHQ, cation NF-QI and neutral NF-Ndef ${ }^{+}$(NF-Ndef); whereas SFR, $g_{\text {iso }}>2.0040$, with anion NF-IQ and radicals NF-SQ. ${ }^{49}$ To gain insight into each radical anisotropy, we convert the theoretical $g$-values into $\Delta g$ and rhombicity and compare them as shown in Fig. 5a. From Fig. 5a, it is possible to see that the species attributed to CCR has $\Delta g<300 \times 10^{-5}$, whereas for SFR, $\Delta g>300 \times 10^{-5}$ with no apparent order for rhombicity. Additionally, based on the spectroscopic values tabulated in Table 1, the radical A-I can be assigned to cationic NF-QI and A-II to neutral NF-Ndef01 and anionic NF-HQ02. On the other hand, species B-I and B-II seem to be related predominantly to species NF-Ndef02 (neutral).

The isotropic $g$ below 2.0035 and the low $\Delta g$ given in Table 1 and Fig. $5 \mathrm{a}$ indicate that the HFEPR signal could be ascribed to only CCR species. Some studies suggest that semiquinone species should present a $g_{x x}$ higher than 2.0055 (Fig. 4 and ref. 72, 76) at the low-field edge of the HFEPR absorption spectra. However, we do not see any $\boldsymbol{g}$ tensor component in Fig. 2. The possible absence of SFR species in melanin is an odd feature at first. However, our result does not imply that NF-SQ does not exist in melanin; it could mean that the experimental condition of the measurement favors the presence of only CCR species. As a matter of fact, we have shown that the melanin pellets result in a higher amount of CCR species. ${ }^{37}$ On top of that, following comproportionation reaction (Scheme 1), it is known that the presence of SQ radicals will dominate the EPR spectra only under alkaline conditions (high pH). ${ }^{34,37,44}$ Hence, 


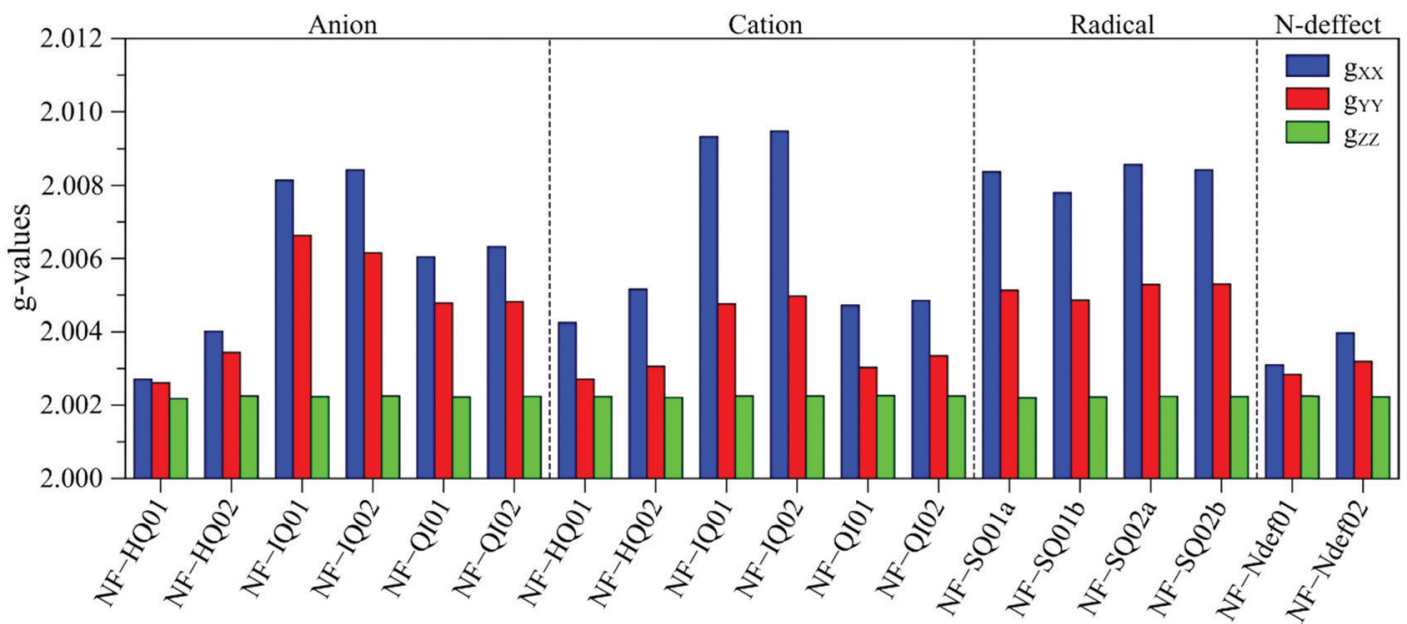

Fig. 4 DFT derived $g$-values for anionic, cationic and radical NF-melanin units. 01 and 02 refer to NF-DHI and NF-DHICA species, respectively.
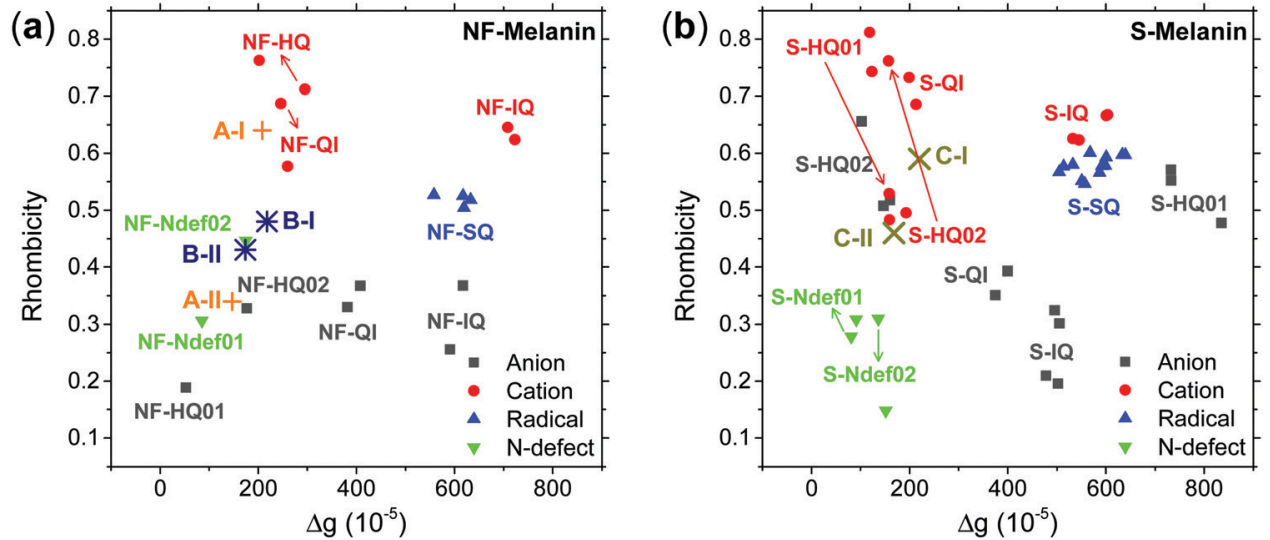

Fig. 5 The plot of the rhombicity as a function of $g$-value anisotropy for the different (a) NF-melanin and (b) S-melanin radical species. The theoretical radicals are represented by the square, circle and triangle symbols; the crosses are each of the experimental radicals. A purely isotropic signal would have $\Delta g=0$ and a completely axial symmetry signal would be obtained with rhombicity equal to 1.0 or 0.0 .

based on the purification process of this synthetic procedure, we also expect that our samples are neutral, which also points towards low SFR content. Such assumption is reinforced with Table S2 (ESI $\dagger$ ) showing that the high-g component decreases or even disappears at neutral and acidic $\mathrm{pH}$. We also note that any effect originated from the temperature treatment during the extraction process does not seem to change the overall paramagnetic species in these melanin samples, as shown in earlier X-band EPR studies, ${ }^{37,38}$ suggesting that we indeed could have a lower amount of SQR species. Another possibility is that the measured signal is already saturated at $0.1 \mathrm{~mW}$ (Fig. S13, ESI $\dagger$ ). In such a case, the measurements should be done at lower microwave power, which is not possible in the spectrometer we used. More studies will be necessary to clarify this behavior.

Now, we turn to sulfonated melanin, i.e., SMel. According to Table 1, the spectroscopic features of SMel are similar to nonfunctionalized melanin, with low isotropic $g$-value $\left(g_{\text {iso }}<\right.$ $2.0035)$ and $\Delta g$ of $220 \times 10^{-5}$ for species C-I and $168 \times 10^{-5}$ for C-II. However, different from NF-melanin that has higher directional dependence at $g_{x x}$ and $g_{y y}$, the C-I component of SMel displayed a low dependence at $g_{y y}$, probably due to the sulfonated groups. In the previous work, we have shown that the free-radical nature of SMel is compatible with NF-melanin. ${ }^{38}$ Hence, the difference in the spectroscopy values may suggest that S-melanin has both the radicals, that is, sulfonated and nonfunctionalized radicals.

The $g$ anisotropy of anionic, cationic and radical S-units was also obtained from DFT and they are displayed in Fig. 6 . Colored spin densities are shown in Fig. S14 and S15 (ESI $\dagger$ ). Similar to NF-melanin, the same $g_{\text {iso }}$ category can be applied for S-melanin, ${ }^{38}$ with the difference that S-HQ01 has an isotropic $g$ higher than 2.0040. Such effect was assigned to a defect from the sulfonated species. ${ }^{38}$ Also, each radical $g$ component shown in Fig. 5b follows a similar order of NF-melanin. A close analysis of Fig. 5b indicates that species C-I is closely related to cationic NF-QI, whereas for C-II anionic to S-HQ-02(a and b) cationic S-HQ01b and S-HQ02(a and c) and neutral NF-Ndef02. 


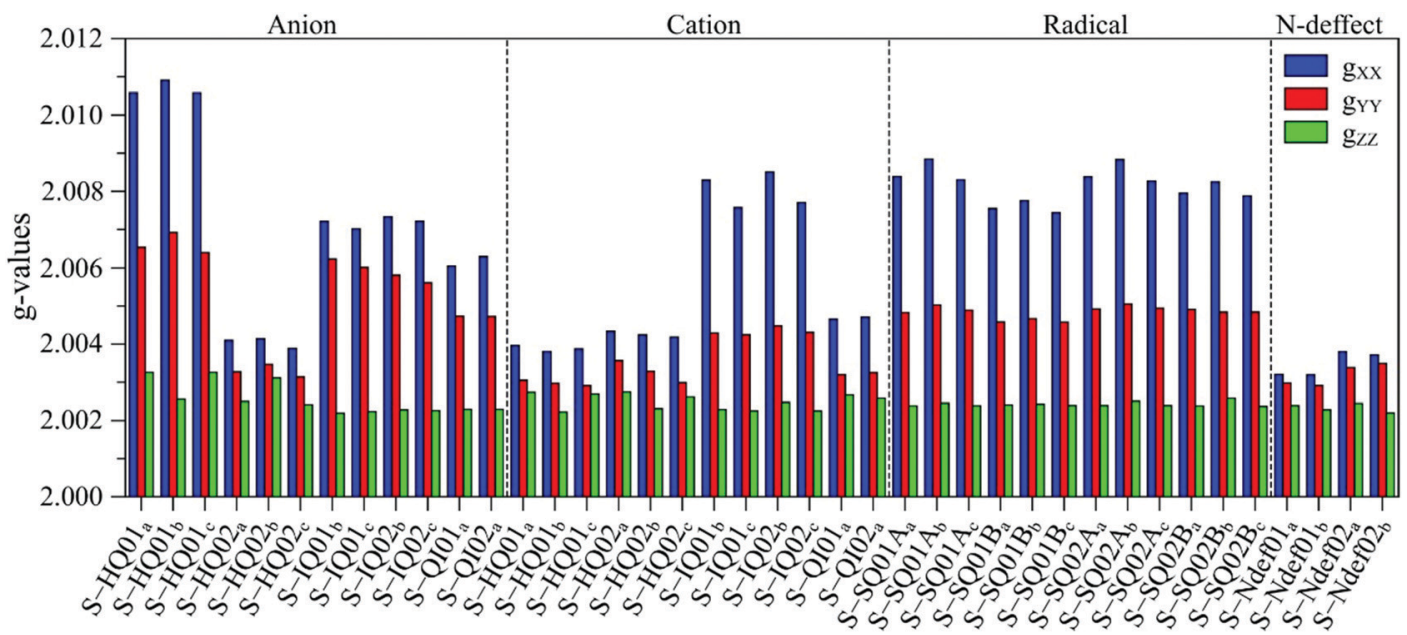

Fig. 6 Anisotropic $g$-value distribution obtained from DFT calculations of anionic, cationic and radical S-melanin monomers. 01 refers to S-DHI and 02 to S-DHICA species.

The spectroscopic similarities in both the groups suggest that SMel can have an equivalent paramagnetic system to NFMel and NFMel-P, consistent with previous X-band analysis. ${ }^{37,38}$

Our HFEPR signal did not show any hyperfine lines related to nitrogen, which would be expected due to the indole structure of melanin (Fig. 1). The suppression of hyperfine lines could be related to exchange narrowing effects, ${ }^{53,67,77}$ as previously shown in organic radicals like 2,2-diphenyl-1-picrylhydrazyl (DPPH). ${ }^{78}$ The exchange is usually associated with a decrease in the inhomogeneous broadening (Table S3, ESI $\dagger$ ) due to large extended spin-spin interaction. ${ }^{77,79}$ However, a previous theoretical study from dimer structures ${ }^{49}$ indicates that their paramagnetic behavior is closely related to the monomers suggesting that the spin is localized on monomers rather than delocalized over many molecular units, potentially hindering the exchange effects. In fact, looking at the spin densities of the most probable units discussed above (Fig. S11, S12 and S14, S15, ESI $\dagger$ ) none of them are centered on the nitrogen. Furthermore, we conducted the dilution ${ }^{77,80}$ and pH-dependent EPR studies on multifrequency continuous-wave/pulsed EPR and pulsed ENDOR experiments to fully understand if the exchange is important or not to melanin EPR spectra. Furthermore, exploring the metal chelation properties ${ }^{39}$ of melanin could also be a promising pathway for future EPR studies.

\section{Conclusion}

In the present work, we have evaluated the free-radical nature of non-functionalized and sulfonated melanin derivatives using high-field/high-frequency EPR $(v=263 \mathrm{GHz})$. Our study confirms the previous analysis that melanin should be viewed as a system with more than one free-radical species. Additionally, it also demonstrates the compatibility of the sulfonated melanin paramagnetic system towards non-functionalized analogs. The simulation of the HFEPR signals suggests the presence of two CCR species, one of them is related to Ndef units, indicating that such species should be considered when analyzing the physicochemistry associated with free radicals. Our results demonstrate the need for more sophisticated analysis to fully unravel the nature of melanin and melanin derivative free radicals.

\section{Conflicts of interest}

The authors declare no competing financial interest.

\section{Acknowledgements}

We thank Prof. Claudio Magon (University of São Paulo) for fruitful discussion and technical support with the baseline and phase correction of the HFEPR spectra. J. V. P., A. B. N. and C. F. O. G. also acknowledge the financial support from São Paulo Research Foundation (FAPESP; grants 12/03116-7, 13/07296-2, 15/ 23000-1), Brazilian Coordination for the Improvement of Higher Education Personnel (CAPES), Brazilian National Council for Scientific and Technological Development (CNPq, grants: 573636/2008-7, 308360/2018-4, 420449/2018-3). The research was also supported by resources supplied by the Center for Scientific Computing (NCC/GridUNESP) of São Paulo State University (UNESP). We also thank the German Ministry of Research and Education (BMBF) for providing financial support through the EPR-Solar network project 03SF0328. B. N. acknowledges the financial support from the DFG (project numbers 410866378 and 410866565).

\section{References}

1 M. Xiao, Y. Li, M. C. Allen, D. D. Deheyn, X. Yue, J. Zhao, N. C. Gianneschi, M. D. Shawkey and A. Dhinojwala, BioInspired Structural Colors Produced via Self-Assembly of Synthetic Melanin Nanoparticles, ACS Nano, 2015, 9, 5454-5460, DOI: 10.1021/acsnano.5b01298. 
2 G. Vitiello, A. Zanfardino, O. Tammaro, M. Di Napoli, M. F. Caso, A. Pezzella, M. Varcamonti, B. Silvestri, G. D'Errico, A. Costantini and G. Luciani, Bioinspired Hybrid Eumelanin-TiO2 Antimicrobial Nanostructures: The Key Role of Organo-Inorganic Frameworks in Tuning Eumelanin's Biocide Action Mechanism through Membrane Interaction, RSC Adv., 2018, 8, 28275-28283, DOI: $10.1039 / \mathrm{c} 8 \mathrm{ra04315a.}$

3 M. D’Ischia, K. Wakamatsu, A. Napolitano, S. Briganti, J.-C. Garcia-Borron, D. Kovacs, P. Meredith, A. Pezzella, M. Picardo, T. Sarna, J. D. Simon and S. Ito, Melanins and Melanogenesis: Methods, Standards, Protocols, Pigm. Cell Melanoma Res., 2013, 26, 616-633, DOI: 10.1111/ pcmr.12121.

4 L. Panzella, G. Gentile, G. D’Errico, N. F. Della Vecchia, M. E. Errico, A. Napolitano, C. Carfagna and M. D'Ischia, Atypical Structural and $\pi$-Electron Features of a Melanin Polymer That Lead to Superior Free-Radical-Scavenging Properties, Angew. Chem., 2013, 52, 12684-12687, DOI: 10.1002/anie.201305747.

5 M. L. Wolbarsht, A. W. Walsh and G. George, Melanin, a Unique Biological Absorber, Appl. Opt., 1981, 20, 2184, DOI: 10.1364/AO.20.002184.

6 A. D. Schweitzer, E. Revskaya, P. Chu, V. Pazo, M. Friedman, J. D. Nosanchuk, S. Cahill, S. Frases, A. Casadevall and E. Dadachova, Melanin-Covered Nanoparticles for Protection of Bone Marrow during Radiation Therapy of Cancer, Int. J. Radiat. Oncol., Biol., Phys., 2010, 78, 1494-1502, DOI: 10.1016/j.ijrobp.2010.02.020.

7 P. Meredith and T. Sarna, The Physical and Chemical Properties of Eumelanin, Pigm. Cell Res., 2006, 19, 572-594, DOI: 10.1111/j.1600-0749.2006.00345.x.

8 M. L. Tran, B. J. Powell and P. Meredith, Chemical and Structural Disorder in Eumelanins: A Possible Explanation for Broadband Absorbance, Biophys. J., 2006, 90, 743-752, DOI: 10.1529/biophysj.105.069096.

9 B. Commoner, J. Townsend and G. E. Pake, Free Radicals in Biological Materials, Nature, 1954, 174, 689-691.

10 M. R. Powell and B. Rosenberg, The Nature of the Charge Carriers in Solvated Biomacromolecules, Bioenergetics, 1970, 1, 493-509, DOI: 10.1007/BF01517187.

11 N. Amdursky, E. D. Głowacki and P. Meredith, Macroscale Biomolecular Electronics and Ionics, Adv. Mater., 2019, 31, 1802221, DOI: 10.1002/adma.201802221.

12 M. Reali, P. Saini and C. Santato, Electronic and Protonic Transport in Bio-Sourced Materials: A New Perspective on Semiconductivity, Mater. Adv. , 2021, 2, 15-31, DOI: 10.1039/ d0ma00579g.

13 H. P. Oliveira, C. F. O. Graeff, C. L. P. S. Zanta, A. C. Galina and P. J. Gonçalves, Synthesis, Characterization and Properties of a Melanin-like/Vanadium Pentoxide Hybrid Compound, J. Mater. Chem., 2000, 10, 371-375, DOI: 10.1039/ a908736b.

14 P. Meredith; B. J. Powell; J. Riesz; R. Vogel; D. Blake; I. Kartini; G. Will and S. Subianto, Broadband PhotonHarvesting Biomolecules for Photovoltaics. In Artificial
Photosynthesis: From Basic Biology to Industrial Application, ed. A. F. Collings and C. Critchley, Wiley-VCH Verlag GmbH \& Co. KgaA, Weinheim, FRG, 2005, pp. 37-65.

15 L. G. S. Albano, J. V. Paulin, L. D. Trino, S. L. Fernandes and C. F. O. Graeff, Ultraviolet-Protective Thin Film Based on PVA-Melanin/Rod-Coated Silver Nanowires and Its Application as a Transparent Capacitor, J. Appl. Polym. Sci., 2019, 136, 47805, DOI: 10.1002/app.47805.

16 R. Xu, A. Gouda, M. F. Caso, F. Soavi and C. Santato, Melanin: A Greener Route To Enhance Energy Storage under Solar Light, ACS Omega, 2019, 4, 12244-12251, DOI: 10.1021/acsomega.9b01039.

17 Z. Tehrani, S. P. Whelan, B. Mostert, J. V. Paulin, M. M. Ali, E. D. Ahmadi, C. F. Graeff, O. de, O. J. Guy and D. T. Gethin, Printable and Flexible Graphene PH Sensors Utilising Thin Film Melanin for Physiological Applications, 2D Mater., 2020, 7, 024008, DOI: 10.1088/2053-1583/ab72d5.

18 M. Ambrico, A. Cardone, T. Ligonzo, V. Augelli, P. F. Ambrico, S. Cicco, G. M. Farinola, M. Filannino, G. Perna and V. Capozzi, Hysteresis-Type Current-Voltage Characteristics in Au/Eumelanin/ITO/Glass Structure: Towards Melanin Based Memory Devices, Org. Electron., 2010, 11, 1809-1814.

19 Y. J. Kim, W. Wu, S. Chun, J. F. Whitacre and C. J. Bettinger, Biologically Derived Melanin Electrodes in Aqueous Sodium-Ion Energy Storage Devices, Proc. Natl. Acad. Sci. U. S. A., 2013, 110, 20912-20917, DOI: 10.1073/ pnas.1314345110.

20 M. Piacenti-Silva, J. C. Fernandes, N. B. de Figueiredo, M. Congiu, M. Mulato and C. F. O. Graeff, Melanin as an Active Layer in Biosensors, AIP Adv., 2014, 4, 037120, DOI: 10.1063/1.4869638.

21 P. Kumar, E. Di Mauro, S. Zhang, A. Pezzella, F. Soavi, C. Santato and F. Cicoira, Melanin-Based Flexible Supercapacitors, J. Mater. Chem. C, 2016, 4, 9516-9525, DOI: 10.1039/c6tc03739a.

22 L. Chang, F. Chen, X. Zhang, T. Kuang, M. Li, J. Hu, J. Shi, L. J. Lee, H. Cheng and Y. Li, Synthetic Melanin E-Ink, ACS Appl. Mater. Interfaces, 2017, 9, 16553-16560, DOI: 10.1021/ acsami.7b03890.

23 L. Migliaccio, S. Aprano, L. Iannuzzi, M. G. Maglione, P. Tassini, C. Minarini, P. Manini and A. Pezzella, Eumelanin-PEDOT:PSS Complementing En Route to MammalianPigment-Based Electrodes: Design and Fabrication of an ITO-Free Organic Light-Emitting Device, Adv. Electron. Mater., 2017, 3, 1600342, DOI: 10.1002/aelm.201600342.

24 L. Migliaccio, M. Gryszel, V. Derek, A. Pezzella and E. D. Głowacki, Aqueous Photo(Electro)Catalysis with Eumelanin Thin Films, Mater. Horiz., 2018, 5, 984-990, DOI: 10.1039/c8mh00715b.

25 M. Sheliakina, A. B. Mostert and P. Meredith, An All-SolidState Biocompatible Ion-to-Electron Transducer for Bioelectronics, Mater. Horiz., 2018, 5, 256-263, DOI: 10.1039/ C7MH00831G.

26 F. G. Cánovas, F. García-Carmona, J. V. Sánchez, J. L. I. Pastor and J. A. L. Teruel, The Role of $\mathrm{PH}$ in the 
Melanin Biosynthesis Pathway, J. Biol. Chem., 1982, 257, 8738-8744.

27 E. S. Bronze-Uhle, A. Batagin-Neto, P. H. P. Xavier, N. I. Fernandes and C. F. O. Graeff, Synthesis and Characterization of Melanin in DMSO, J. Mol. Struct., 2013, 1047, 102-108, DOI: 10.1016/j.molstruc.2013.04.061.

28 M. S. Blois, A. B. Zahlan and J. E. Maling, Electron Spin Resonance Studies on Melanin, Biophys. J., 1964, 4, 471-490, DOI: 10.1016/s0006-3495(64)86797-7.

29 F. J. Grady and D. C. Borg, Electron Paramagnetic Resonance Studies on Melanins. I. The Effect of PH on Spectra at Q Band, J. Am. Chem. Soc., 1968, 90, 2949-2952, DOI: 10.1021/ja01013a036.

30 T. Properties Sarna, and Function of the Ocular Melanin - a Photobiophysical View, J. Photochem. Photobiol., B, 1992, 12, 215-258, DOI: 10.1016/1011-1344(92)85027-R.

31 R. Dunford, E. J. Land, M. Rozanowska, T. Sarna and T. G. Truscott, Interaction of Melanin with Carbon- and Oxygen-Centered Radicals from Methanol and Ethanol, Free Radical Biol. Med., 1995, 19, 735-740, DOI: 10.1016/08915849(95)00059-7.

32 K. J. Reszka, Z. Matuszak and C. F. Chignell, Lactoperoxidase-Catalyzed Oxidation of Melanin by Reactive Nitrogen Species Derived From Nitrite $\left(\mathrm{NO}_{2}{ }^{-}\right)$: An EPR Study, Free Radical Biol. Med., 1998, 25, 208-216, DOI: 10.1016/S0891-5849(98)00058-6.

33 L. Najder-Kozdrowska, B. Pilawa, A. B. Więckowski, E. Buszman and D. Wrześniok, Influence of Copper(II) Ions on Radicals in DOPA-Melanin, Appl. Magn. Reson., 2009, 36, 81-88, DOI: 10.1007/s00723-009-0001-y.

34 A. B. Mostert, G. R. Hanson, T. Sarna, I. R. Gentle, B. J. Powell and P. Meredith, Hydration-Controlled X-Band EPR Spectroscopy: A Tool for Unravelling the Complexities of the Solid-State Free Radical in Eumelanin, J. Phys. Chem. $B$, 2013, 117, 4965-4972, DOI: 10.1021/jp401615e.

35 S. B. Rienecker, A. B. Mostert, G. Schenk, G. R. Hanson and P. Meredith, Heavy Water as a Probe of the Free Radical Nature and Electrical Conductivity of Melanin, J. Phys. Chem. B, 2015, 119, 14994-15000, DOI: 10.1021/ acs.jpcb.5b08970.

36 A. B. Mostert, S. B. Rienecker, C. Noble, G. R. Hanson and P. Meredith, The Photoreactive Free Radical in Eumelanin, Sci. Adv., 2018, 4, eaaq1293, DOI: 10.1126/sciadv.aaq1293.

37 J. V. Paulin, A. Batagin-Neto and C. F. O. Graeff, Identification of Common Resonant Lines in the EPR Spectra of Melanins, J. Phys. Chem. B, 2019, 123, 1248-1255, DOI: 10.1021/acs.jpcb.8b09694.

38 J. V. Paulin, A. Batagin-Neto, P. Meredith, C. F. O. Graeff and A. B. Mostert, Shedding Light on the Free Radical Nature of Sulfonated Melanins, J. Phys. Chem. B, 2020, 124, 10365-10373, DOI: 10.1021/acs.jpcb.0c08097.

39 C. C. Felix, J. S. Hyde, T. Sarna and R. C. Sealy, Interactions of Melanin with Metal Ions. Electron Spin Resonance Evidence for Chelate Complexes of Metal Ions with Free Radicals, J. Am. Chem. Soc., 1978, 100, 3922-3926, DOI: 10.1021/ja00480a044.
40 C. C. Felix, J. S. Hyde and R. C. Sealy, Photoreactions of Melanin: A New Transient Species and Evidence for Triplet State Involvement, Biochem. Biophys. Res. Commun., 1979, 88, 456-461, DOI: 10.1016/0006-291X(79)92070-9.

41 W. Froncisz, T. Sarna and J. S. Hyde, $\mathrm{Cu}^{2+}$ Probe of MetalIon Binding Sites in Melanin Using Electron Paramagnetic Resonance Spectroscopy, Arch. Biochem. Biophys., 1980, 202, 289-303, DOI: 10.1016/0003-9861(80)90430-0.

42 R. C. Sealy; C. C. Felix; J. S. Hyde and H. M. Swartz Structure and Reactivity of Melanins: Influence of Free Radicals and Metal Ions. In Free Radicals in Biology, Academic Press, 1980; pp. 209-259, DOI: 10.1016/B978-0-12-566504-9.50014-3.

43 S. Chio, J. S. Hyde and R. C. Sealy, Temperature-Dependent Paramagnetism in Melanin Polymers, Arch. Biochem. Biophys., 1980, 199, 133-139, DOI: 10.1016/00039861(80)90265-9.

44 S. Chio, J. S. Hyde and R. C. Sealy, Paramagnetism in Melanins: PH Dependence, Arch. Biochem. Biophys., 1982, 215(1), 100-106, DOI: 10.1016/0003-9861(82)90283-1.

45 T. Sarna and R. C. Sealy, Free Radicals from Eumelanins: Quantum Yields and Wavelength Dependence, Arch. Biochem. Biophys., 1984, 232, 574-578, DOI: 10.1016/00039861(84)90575-7.

46 M. Pasenkiewicz-Gierula and R. C. Sealy, Analysis of the ESR Spectrum of Synthetic Dopa Melanin, Biochim. Biophys. Acta, 1986, 884, 510-516, DOI: 10.1016/0304-4165(86)90202-3.

47 T. Sarna and P. M. Plonka Biophysical Studies of Melanin: Paramagnetic, Ion-Exchange and Redox Properties of Melanin Pigments and Their Photoreactivity. In Biomedical EPR, Part A: Free Radicals, Metals, Medicine, and Physiology, ed., S. S. Eaton, G. R. Eaton and L. J. Berliner, Springer US, 2005; pp. 125-146, DOI: 10.1007/0-387-26741-7_7.

48 T. Sarna and H. A. Swartz The Physical Properties of Melanins. In The Pigmentary System: Physiology and Pathophysiology, ed., Nordlund, J. J., Boissy, R. E., Hearing, V. J., King, R. A., Oetting, W. S. and Ortonne, J., Blackwell Publishing, 2006, pp. 191-212.

49 A. Batagin-Neto, E. S. Bronze-Uhle, C. F. Graeff and O. de, Electronic Structure Calculations of ESR Parameters of Melanin Units, Phys. Chem. Chem. Phys., 2015, 17, 7264-7274, DOI: 10.1039/c4cp05256k.

50 G. R. Eaton and S. S. Eaton, High-Field and High-Frequency EPR, Appl. Magn. Reson., 1999, 16, 161-166, DOI: 10.1007/ bf03161931.

51 M. Bennati and T. F. Prisner, New Developments in High Field Electron Paramagnetic Resonance with Applications in Structural Biology, Reports Prog. Phys., 2005, 68, 411-448, DOI: 10.1088/0034-4885/68/2/R05.

52 K. Möbius and A. Savitsky High-Field EPR Spectroscopy on Proteins and Their Model Systems, Royal Society of Chemistry, 2008, DOI: 10.1039/9781847559272.

53 M. M. Roessler and E. Salvadori, Principles and Applications of EPR Spectroscopy in the Chemical Sciences, Chem. Soc. Rev., 2018, 47, 2534-2553, DOI: 10.1039/c6cs00565a.

54 J. Wünsche, F. Cicoira, C. F. O. Graeff and C. Santato, Eumelanin Thin Films: Solution-Processing, Growth, and 
Charge Transport Properties, J. Mater. Chem. B, 2013, 1, 3836-3842, DOI: 10.1039/c3tb20630k.

55 M. Piacenti-Silva, A. A. Matos, J. V. Paulin, R. A. Alavarce, S. da, R. C. de Oliveira and C. F. Graeff, Biocompatibility Investigations of Synthetic Melanin and Melanin Analogue for Application in Bioelectronics, Polym. Int., 2016, 65, 1347-1354, DOI: 10.1002/pi.5192.

56 L. G. S. Albano, E. Di Mauro, P. Kumar, F. Cicoira, C. F. O. Graeff and C. Santato, Novel Insights on the Physicochemical Properties of Eumelanins and Their DMSO Derivatives, Polym. Int., 2016, 65, 1315-1322, DOI: 10.1002/ pi.5167.

57 J. V. Paulin, A. G. Veiga, Y. Garcia-Basabe, M. L. M. Rocco and C. F. Graeff, Structural and Optical Properties of Soluble Melanin Analogues with Enhanced Photoluminescence Quantum Efficiency, Polym. Int., 2018, 67, 550-556, DOI: 10.1002/pi.5543.

58 E. S. Bronze-Uhle, J. V. Paulin, M. Piacenti-Silva, C. Battocchio, M. L. M. Rocco, C. F. Graeff and O. de, Melanin Synthesis under Oxygen Pressure, Polym. Int., 2016, 65, 1339-1346, DOI: 10.1002/pi.5185.

59 S. N. Dezidério, C. A. Brunello, M. I. N. da Silva, M. A. Cotta and C. F. O. Graeff, Thin Films of Synthetic Melanin, J. Non. Cryst. Solids, 2004, 338-340, 634-638, DOI: 10.1016/ j.jnoncrysol.2004.03.058.

60 J. J. Wittmann, T. V. Can, M. Eckardt, W. Harneit, R. G. Griffin and B. Corzilius, High-Precision Measurement of the Electron Spin $\mathrm{g}$ Factor of Trapped Atomic Nitrogen in the Endohedral Fullerene N@C60, J. Magn. Reson., 2018, 290, 12-17, DOI: 10.1016/j.jmr.2018.02.019.

61 K. A. Earle, D. E. Budil and J. H. Freed, 250-GHz EPR of Nitroxides in the Slow-Motional Regime: Models of Rotational Diffusion, J. Phys. Chem., 1993, 97, 13289-13297, DOI: 10.1021/j100152a037.

62 S. Stoll and A. Schweiger, EasySpin, a Comprehensive Software Package for Spectral Simulation and Analysis in EPR, J. Magn. Reson., 2006, 178, 42-55, DOI: 10.1016/j.jmr.2005.08.013.

63 J. J. P. Stewart MOPAC 2016 http://openmopac.net/ MOPAC2016.html.

64 M. J. Frisch, G. W. Trucks, H. B. Schlegel, G. E. Scuseria, M. Robb, J. Cheeseman, G. Scalmani, V. Barone, B. Mennucci, G. A. H. Petersson, H. Nakatsuji, M. Caricato, X. Li, H. P. Hratchian, A. F. Izmaylov, J. Bloino, G. Zheng, J. L. Sonnenberg, M. Hada and D. Fox, Gaussian 09, 2009.

65 M. W. Schmidt, K. K. Baldridge, J. A. Boatz, S. T. Elbert, M. S. Gordon, J. H. Jensen, S. Koseki, N. Matsunaga, K. A. Nguyen, S. Su, T. L. Windus, M. Dupuis and J. A. Montgomery, General Atomic and Molecular Electronic Structure System, J. Comput. Chem., 1993, 14, 1347-1363, DOI: $10.1002 /$ jcc.540141112.

66 J. A. Weil and J. R. Bolton Electron Paramagnetic Resonance: Elementary Theory and Practical Applications, Wiley: New York, 2nd ed., 2007.

67 C. P. Poole Electron Spin Resonance: A Comprehensive Treatise on Experimental Techniques, John Wiley \& Sons, New York, 1983.
68 M. B. Clark, J. A. Gardella, T. M. Schultz, D. G. Patil and L. Salvati, Solid-State Analysis of Eumelanin Biopolymers by Electron Spectroscopy for Chemical Analysis, Anal. Chem., 1990, 62, 949-956, DOI: 10.1021/ac00208a011.

69 F. Bernsmann, A. Ponche, C. Ringwald, J. Hemmerle, J. Raya, B. Bechinger, J. C. Voegel, P. Schaaf and V. Ball, Characterization of Dopamine-Melanin Growth on Silicon Oxide, J. Phys. Chem. C, 2009, 113, 8234-8242, DOI: 10.1021/ jp901188h.

70 F. Neese, The ORCA Program System, Wiley Interdiscip. Rev.: Comput. Mol. Sci., 2012, 2, 73-78, DOI: 10.1002/wcms.81.

71 I. Davis, T. Koto, J. R. Terrell, A. Kozhanov, J. Krzystek and A. Liu, High-Frequency/High-Field Electron Paramagnetic Resonance and Theoretical Studies of Tryptophan-Based Radicals, J. Phys. Chem. A, 2018, 122, 3170-3176, DOI: 10.1021/acs.jpca.7b12434.

72 D. S. Tipikin, S. G. Swarts, J. W. Sidabras, F. Trompier and H. M. Swartz, Possible Nature of the Radiation-Induced Signal in Nails: High-Field EPR, Confirming Chemical Synthesis, and Quantum Chemical Calculations, Radiat. Prot. Dosim., 2016, 172, 112-120, DOI: 10.1093/rpd/ncw216.

73 M. R. Fuchs, A. Schnegg, M. Plato, C. Schulz, F. Müh, W. Lubitz and K. Möbius, The Primary Donor Cation P +. in Photosynthetic Reaction Centers of Site-Directed Mutants of Rhodobacter Sphaeroides: G-Tensor Shifts Revealed by High-Field EPR at $360 \mathrm{GHz} / 12.8$ T, Chem. Phys., 2003, 294, 371-384, DOI: 10.1016/S0301-0104(03)00319-7.

74 A. J. Stone, Gauge Invariance of the $\mathrm{g}$ Tensor, Proc. $R$. Soc. London, Ser. A, 1963, 271, 424-434, DOI: 10.1098/rspa.1963.0027.

75 A. J. G. Stone, Factors of Aromatic Free Radicals, Mol. Phys., 1963, 6, 509-515, DOI: 10.1080/00268976300100571.

76 C. Bährle, T. U. Nick, M. Bennati, G. Jeschke and F. Vogel, High-Field Electron Paramagnetic Resonance and Density Functional Theory Study of Stable Organic Radicals in Lignin: Influence of the Extraction Process, Botanical Origin, and Protonation Reactions on the Radical g Tensor, J. Phys. Chem. A, 2015, 119, 6475-6482, DOI: 10.1021/ acs.jpca.5b02200.

77 S. K. Misra and S. Diehl, Theory of EPR Lineshape in Samples Concentrated in Paramagnetic Spins: Effect of Enhanced Internal Magnetic Field on High-Field HighFrequency (HFHF) EPR Lineshape, J. Magn. Reson., 2012, 219, 53-60, DOI: 10.1016/j.jmr.2012.03.023.

78 J. Krzystek, A. Sienkiewicz, L. Pardi and L. C. Brunel, DPPH as a Standard for High-Field EPR, J. Magn. Reson., 1997, 125, 207-211, DOI: 10.1006/jmre.1996.1098.

79 J. Han, H. Zhang and D. Abramavicius, Exchange Narrowing and Exciton Delocalization in Disordered J Aggregates: Simulated Peak Shapes in the Two Dimensional Spectra, J. Chem. Phys., 2013, 139, 034313, DOI: 10.1063/1.4812927.

80 T. Rodrigues, C. G. Dos Santos, A. Riposati, L. R. S. Barbosa, P. Di Mascio, R. Itri, M. S. Baptista, O. R. Nascimento and I. I. Nantes, Photochemically Generated Stable Cation Radical of Phenothiazine Aggregates in Mildly Acid Buffered Solutions, J. Phys. Chem. B, 2006, 110, 12257-12265, DOI: 10.1021/jp0605404. 